\title{
An Approach to Calculate Overall Efficiency of Rolling Stock for an Urban Rail Transit System
}

Qamar Mahboob, Technische Universität Dresden \& Pakistan Railways

Thomas Stoiber, Ernst Basler + Partner AG

Stephanie Gottstein, Stadtwerke München $\mathrm{GmbH}$

Antonios Tsakarestos, Technische Universität München

\begin{abstract}
Inefficiency of urban rail transit systems (URTS) results in a lower standard of service than required and has negative economic impacts. Many efforts have been undertaken to calculate the efficiency of URTS or their subunits. Overall equipment efficiency (OEE) as a measure of efficiency is a simple, flexible, and efficient formula. The OEE measure ensures that equipment is available and fully capable of producing quality goods/services for the maximum time of operation and is being used in a proper way. This paper discusses the idea of the OEE from the field of production and how it can be applied to a URTS. Overall efficiency of rolling stock (RS) is a major influencing factor on the efficiency of URTS. Therefore, the three fundamental parameters of OEE - availability, performance, and service quality-are defined for the RS of a URTS, and a method for their calculation is presented. The usefulness of OEE for URTS is investigated.
\end{abstract}

\section{Introduction}

Improving and maintaining the efficiency of rail transit systems has become important due to limited public funds and an increase in transport demand (Sulek 
et al. 2000). There are many ways to calculate the efficiency of a public transport system or one of its subunits. For schedule and operating personnel efficiencies of a transit system, we refer to Vuchic (2005) and Edwards (1992). In terms of economics, technical efficiency is measured by the ratio of output to input (Cooper et al. 2004). Depending on the type of system, there can be single or multiple inputs/ outputs. In the case of an urban rail transit system (URTS), multiple outputs are produced by multiple inputs. Currently, several inputs/outputs of a transit system are in use to develop a performance measurement system (Sulek and Lind 2000) . Additionally, each URTS has different subunits, with each subunit having a variety of inputs/outputs. Individual lines of network, transit units of a particular type in a vehicle fleet, parking management, park-and-ride lots, and collection of fares are some examples of subunits. Any subunit of a URTS that runs with low efficiency can cause the following:

- less profit for the transport company

- cost increases due to extra wages for drivers and maintenance and other staff who are in charge of overcoming actual deficiencies

- increase in service costs because of rework

- waste of energy, materials, and work force

- impacts on pricing

Therefore, the focus should be on improving the overall efficiency of URTS. One way of achieving this objective is to identify and improve the efficiencies of the individual subunits (Barnum et al. 2007). Another way to improve would be to adopt strategies that result in the more efficient use of transportation resources (VTPI 2011), which is called transport demand management. It is important that different measures for strategy and input/output for efficiency must correspond to the objectives of the transit agency. However, the core objective of any transit agency is to ensure that the transit system is available, fully capable of producing quality services for the maximum time of operation, and being used properly. This leads us to use a measure that is called overall equipment efficiency (OEE) and is taken from the field of production (Borris 2006). It focuses on the core objective mentioned above. OEE as a measure for efficiency calculation is a simple, effective, and flexible formula. It measures the efficiency of equipment during its planned operation time.

This paper outlines the background of OEE and describes how it can be defined for URTS. Some issues regarding its application to URTS are addressed. It is investigated whether the OEE measure is useful for calculating the overall efficiency of URTS. What kind of information and results will be obtained for the specific case 
of URTS if we apply this measure? The rolling stock (RS) is a subunit of URTS and serves as the backbone of any system. To simplify the study, we applied the OEE measure to the RS. In this work, the RS of the Munich URTS was chosen as the real-world application. OEE is defined and calculated by defining and calculating the three parameters-availability, performance, and quality yield_for the rolling stock of the URTS.

\section{Theoretical Background}

The overall efficiency of a transit system can be calculated by correctly identifying subunit efficiencies. Each subunit has specific inputs/outputs. For example, Barnum et al. (2007) show that the key outputs for park-and-ride lots of the Chicago Transit Authority are (1) number of parked cars, as a proxy for number of passenger trips, and (2) parking revenues. The key inputs are (1) number of parking spaces and (2) operating expenses. To obtain a comprehensive efficiency measure for comparing the parking lots, each lot's outputs and inputs are aggregated with a relevant weighting scheme, and then the aggregated outputs are divided by the aggregated inputs; see Eq. (1):

$$
\text { Efficiency }=\frac{\text { Output Weight } 1 \cdot \text { Output } 1+\text { OutputWeight } 2 \cdot \text { Output } 2}{\text { Input Weight } 1 \cdot \text { Input } 1+\text { Input Weight } 2 \cdot \text { Input } 2}
$$

The same weights are applied to the inputs and outputs of each parking lot, and then the resulting values are compared. However, one must keep in mind that the weighting scheme is completely dependent on the goals and objectives of the transit authority. Schedule efficiency is one of the important measures of operating efficiency (Edwards 1992). It is defined as the ratio of the sum of operating time (in two directions) to cycle time. Basically, it reflects the terminal time losses. Operating (or travel) time $T_{0}$ is the scheduled time interval between the departure of a transit unit (TU) from one terminal and its arrival at another. The operating time is, therefore, the sum of station-to-station travel times for all $i$ interstation spacings between terminals.

$$
T_{0}=\sum_{i} T_{s_{i}}=\sum_{i}\left(t_{r_{i}}+t_{s_{i}}\right)
$$

Where, the station-to-station time $T_{\mathrm{S}}$ is the time interval between a TU's departures from two neighboring stations; it is equal to the running time $t_{r}$ plus the station standing time $t_{s}$ on any spacing $i$. 


$$
T_{s_{i}}=t_{r_{i}}+t_{s_{i}}
$$

Terminal time $t_{t}$ is the time a TU spends at a line terminal. This time is provided for vehicle turning or change of driver's cab, resting of the crew, adjustment of sched$u l e$ and recovery of delays incurred in travel. The terminal time $t_{t}$ is determined as the percentage of operating time on line (Vuchic 2005). This is minimized in automatic train operation where there is no need for driver breaks, turning of vehicles, etc. Cycle time $T$ is the total round trip on line, or the interval between the two consecutive times a TU (in regular service) leaves the same terminal. It consists of operating times for the two directions and terminal times.

$$
T=2\left(T_{0}+t_{t}\right)
$$

Another way of calculating the cycle time is to multiply the number of transit units, $N_{T U}$, with the headway (time interval in minutes between the moments when two successive TUs pass a fixed point on a transit line in the same direction) $h$. Integer values that are equal to or greater than the computed value are taken for $T, N_{T U}$ and $h$. Finally, the coefficient of schedule efficiency $\eta_{t}$ becomes

$$
\eta_{t}=\frac{2 T_{0}}{T}=\frac{\sum_{i}\left(t_{r i}+t_{s i}\right)}{2\left(T_{0}+t_{t}\right)}
$$

Another method of efficiency calculation is to use the overall equipment efficiency $(\mathrm{OEE})$. It is a way of calculating the percentage of actual effectiveness of the equipment that is consuming inputs for some outputs (Borris 2006).

\section{Overall Equipment Efficiency}

All equipment is intended to produce some kind of output. The type of equipment and its inputs and design limitations will determine how much output can be produced per unit time. For example, scheduled maintenance during the service life of equipment is one of the inputs that affects the output rate and the quality of the outcome. The output can be affected by the following failures:

- The equipment breaks down completely: This is when the equipment produces no products (i.e., goods or services) at all. This is known as a total failure. In this case, times without operation/production may help diagnose the failure easily.

- The equipment still produces products but it lost speed: The equipment is running below its capacity and working slower than under normal operating conditions. 
- The equipment may reduce the quality of the product: Quality can be affected in several ways. One way is that the equipment's outputs do not meet customer demands or market standards and are rejected. Quality problems result in a loss of profit.

The aim of the OEE measure is to ensure that equipment is available and fully capable of producing quality products for the maximum time of production and is used properly. To ensure this, OEE as a measure of efficiency is been defined as the product of (1) availability, (2) performance, and (3) quality yield of the equipment (Borris 2006; McCarthy 2001). A brief description of each parameter is given below.

\section{Availability of Equipment}

The equipment is not capable of producing goods or services if it is down for scheduled or unscheduled maintenance. Availability of the equipment is the ratio of the amount of time that the equipment is capable of producing quality product to the total time it could be running.

$$
\text { Availability }(\%)=\frac{\text { Total time available }- \text { Downtime }}{\text { Total time available }} \cdot 100
$$

There are two types of downtime-planned and unplanned. Usually, planned downtime is adopted in accordance with manufacturer standards. At some point, planned downtime becomes purely dependent on the running time of the equipment, maintenance standards, and local conditions (Dhillon 2002). Unplanned downtime arises due to various uncertainties in the design, operation, maintenance, and environment of the equipment that affects the availability of the equipment.

\section{Performance of Equipment}

If the equipment is running at a speed lower than its capacity, this causes a loss of production. Equipment that is running at half its normal speed is equivalent to 50 percent downtime. Therefore, the performance of equipment is defined with reference to production. It is the ratio of the amount of the manufactured products (for a given production uptime) to the amount of products that could have been manufactured.

$$
\text { Performance }(\%)=\frac{\text { Number of units produced }}{\text { Possible number of units }} \cdot 100
$$

\section{Quality Yield of Equipment}

Generally, quality is conformance to requirements or the degree to which a produced unit, function, or process satisfies the needs of users (Omdahl 1988). It is the 
intention of equipment owner to constantly produce accurate products that users need. Rejection or failure of the product often causes unavailability of the equipment. For example, when equipment produces substandard products, more testing has to be done to fix the problem. This implies that the availability of the equipment is also affected. Additionally, any equipment with usability of less than 100 percent is rarely accepted. Therefore, Borris (2006) and McCarthy (2001) define the quality yield of the product as the ratio of the amount of acceptable products made to the total amount of products made (including any unacceptable products).

$$
\text { Quality yield }(\%)=\frac{\text { Number of units produced }- \text { Number of defective units }}{\text { Number of units produced }} \cdot 100
$$

Finally, the OEE becomes

$$
\text { OEE }=\text { Availability } \cdot \text { Performance } \cdot \text { Quality Yield }
$$

From the data in Table 1, it can be clearly seen that even if the equipment has no downtime (100\% availability), the OEE can still be unacceptably low because of the losses of performance and quality yield. With no downtime and both performance and quality reduced to 50 percent, the resultant OEE falls to a value as low as 25 percent. This illustrates how the parameters are correlated and a loss in any of the three parameters can drastically affect the OEE, which is the aim of OEE measure.

Table 1. Effects of Parameters of OEE

\begin{tabular}{cccc}
\hline Availability & Performance & Quality Yield & OEE \\
\hline $100 \%$ & $100 \%$ & $100 \%$ & $100 \%$ \\
$100 \%$ & $50 \%$ & $50 \%$ & $25 \%$ \\
$100 \%$ & $100 \%$ & $50 \%$ & $50 \%$ \\
$75 \%$ & $75 \%$ & $75 \%$ & $42.18 \%$ \\
$50 \%$ & $50 \%$ & $50 \%$ & $12.5 \%$ \\
\hline
\end{tabular}

\section{Overall Efficiency of Rolling Stock}

OEE as a measure of calculating efficiency is a simple, effective, and flexible formula. It measures the efficiency of the equipment during its planned operation time; planned downtime does not affect OEE. This motivates an investigation into the use of the OEE measure for calculating the overall efficiency of URTS to ensure that a URTS is available, has full operational capability for the maximum time of operation, and is being used properly. This approach can be applied to any individual transit line or extended to a complete URTS. Since it is not possible to discuss the overall efficiency of a complete URTS, our study is restricted to the rolling stock 
(RS) that is a vital subunit of a URTS. Hence, OEE parameters are considered for the rolling stock of a URTS.

\section{Availability of Rolling Stock}

The availability of RS corresponds to the number of TUs that are available for service each day. When a transit unit remains in the maintenance workshop/depot for extra (i.e., other than standard) inspections and checkups and can therefore not be put into service, this is regarded as an availability problem. The maintenance department plans how many hours each transit unit has to spend for checkups and other maintenance per unit time. It implies that TUs should be available for operation in periods other than that reserved for standard maintenance work and checkups. In case of extra inspections and maintenance, the total time available for operation will be less than usual. Hence, availability of the RS can be defined as the ratio of the amount of time that the TUs are capable of producing quality services to the total time they could be running.

$$
\text { Availability }(\%)=\frac{\text { Total time available for operation }}{\text { Schedule time }} \cdot 100
$$

When a TU is out of service due to unforeseen and unavoidable reasons, it causes unplanned downtime. The total time available for operation is the difference between scheduled time and unplanned downtime. It can be calculated by multiplying the total number of TUs available for service (and ready to be put in service at any time) within a transit agency and the number of service hours per day. Scheduled time is the difference between total time and planned downtime. Normally, it depends on the number of years a TU has been in use, the care and maintenance history of a TU, the complexity and variety of the components used in the unit, and the time required for the individual planned activity. A way of deciding to go ahead with a planned downtime activity is described by Levitt (1997). The maximum preventive maintenance time required to carry out a given percentage of all scheduled preventive maintenance actions is given in Dhillon (2002).

\section{Performance of Rolling Stock}

Performance of the rolling stock is defined with respect to its output. One of the key RS outputs is the number of kilometers traveled per unit time. More kilometers per unit time offers more spaces per unit time; thus, ridership can be increased and more revenue is generated. A fault in a component of the TU can reduce its speed and cause it to run at a speed lower than its capability. If fewer kilometers per unit time than planned are caused by slower TUs, this is a performance loss. In other 
words, a transit unit running at half speed is the same as having 50 percent downtime for a particular period of time. However, in case of performance losses, passengers remain in the system, and the financial result would not be worst. Keeping in view the above facts, we can define the performance of the rolling stock as the ratio of actual kilometers (after considering the time loss) and standard kilometers (when there is no time loss due to any failure).

$$
\text { Performance }(\%)=\frac{\text { Actual kilometers }}{\text { Standard kilometers }} \cdot 100
$$

Where, actual kilometers $=$ standard kilometers - kilometers lost; standard kilometers $=N_{T U} \cdot N \cdot$ average distance per trip; $N=$ total number of (round) trips (per unit time) completed by the individual TU. The number of TUs, $N_{T U}$, can be calculated as discussed in Section 2.

Any loss of kilometers (during operation) can occur due to a variety of problems in the RS components. These problems may not appear during the scheduled maintenance in the workshop. This implies that the performance of the RS is the function of the operating time when the RS is performing its intended function. In other words, the performance of the RS cannot be judged unless we put it into service. Operational data of transit units can be analyzed to determine the kilometers lost. One way of calculating these kilometer losses is to calculate the loss of cycle time. In this way, the percentage loss of cycle time represents the percentage loss of kilometers for the respective trip.

\section{Quality Yield of Rolling Stock}

Based on different user, owner, and stakeholder expectations, the quality of the rolling stock can be defined in many ways. In reference to the quality yield described previously, a complete failure of one of the TUs during operation is regarded as a quality issue. In case of a complete TU failure, passengers are forced to leave the TU. Additionally, a complete TU failure (during operation) has the potential to prevent other TUs from moving into the network. This kind of service usability is rarely accepted by passengers and can lead to rejection of the service. Here, financial results may be worse because it is more likely that passengers will leave the system. In this respect, the quality of the RS can be defined as the ratio of the transit units actually working during operation time without failure to the total of maintained units put into service.

$$
\text { Quality }(\%)=\frac{\text { Number of } T . U \text { worked }}{\text { Total T. } U \text { in service }} \cdot 100
$$


Where, number of cars worked $=$ total of cars put in service - cars failed during service.

It is important to note that losses of kilometers are not taken into account here, as they are relevant to performance issues.

\section{Overall Efficiency of Rolling Stock of Urban Rail Transit System in Munich}

The RS of the URTS in Munich has three types. The A-type was introduced in 1972 and has 360 cars (one TU contains six cars), which constitutes 62 percent of the total RS. The B-type was introduced in 1981 and has 114 cars, which is 19.5 percent of the total RS. The C-type was introduced in 2002 and has 108 cars, which is 18.5 percent of the total RS. As a result, the total number of cars that the Munich public transport authority (MVG) has available is 582. However, the maximum number of cars required during peak hour periods does not exceed 470 .

To show the application of the OEE measure, we considered the data for only one hour (4 PM to 5 PM) of every Monday in the year 2008. The reason for choosing this particular interval is that there is maximum utilization of the RS during this hour. Additionally, one hour of data is easy to handle to calculate the distance traveled by TUs during operation time, cycle time, headway time for all TUs, and number of TUs for different headways, distance per trip, total trips, and failed TUs for the specified period. Taking the previous definitions as a basis, the three parameters of OEE for the RS are calculated in the following way:

$$
\begin{aligned}
& \text { Availability }=\left(0.62 \cdot \sum_{\mathrm{a}=1}^{360} \frac{\mathrm{Tsch}(\mathrm{a})-\mathrm{Tup}(\mathrm{a})}{\mathrm{Tt}(\mathrm{a})-\mathrm{Tpl}(\mathrm{a})}+0.195 \cdot \sum_{\mathrm{b}=1}^{114} \frac{\mathrm{Tsch}(\mathrm{b})-\mathrm{Tup}(\mathrm{b})}{\mathrm{Tt}(\mathrm{b})-\mathrm{Tpl}(\mathrm{b})}+0.185\right. \\
& \left.\quad \sum_{\mathrm{c}=1}^{108} \frac{\operatorname{Tsch}(\mathrm{c})-\operatorname{Tup}(\mathrm{c})}{\mathrm{Tt}(\mathrm{c})-\operatorname{Tpl}(\mathrm{c})}\right) \cdot 100
\end{aligned}
$$

Where, the lower-case letters $a, b$, and c represent the three types of rolling stock A, B and C; the weightings $0.62,0.195$ and 0.185 are the percentages of each type of rolling stock in the whole fleet; Tsch = scheduled time for operation; $\mathrm{Tsch}=\mathrm{Tt}$ $\mathrm{Tpl}, \mathrm{Tt}=$ total time for operation; Tup = unplanned downtime; and $\mathrm{Tpl}=$ planned downtime. After inserting the values into the equation, we get availability: 


$$
\begin{aligned}
\text { Availability } & =\left[\left(\frac{0.62(7710-557)}{7810-110}\right)+\left(\frac{0.195(2376-286)}{2398-22}\right)+\left(\frac{0.185(2332-442.5)}{2354-2}\right)\right] \cdot 100 \\
& =89.58 \%
\end{aligned}
$$

To obtain the performance of the URTS, it is necessary to calculate the number of TUs in operation, the total trips covered by all TUs, and the average distance covered per trip. Since the URTS in Munich has six underground lines, $\left(U_{1}, \ldots, U_{6}\right)$ $T, N_{T \cdot U}$, and $h$ were calculated for each line according to the formulas given earlier. Finally,

$$
\text { Total trips by all T. Us }=\sum_{U}\left(\frac{T}{h} \cdot N\right)=66.07, \quad U=1,2, \ldots, 6
$$

The geographical map of the transit network is used as the basis to calculate the distance traveled by each TU for an individual line. The average distance per trip (for all TU of the six lines) is, thus, the standard kilometers that the RS could run. Depending on the number of the different types of TUs introduced into service, the $A, B$ and $C$ types could run 1442.19 , , and $\mathrm{km}$, respectively. The actual number of kilometers traveled by all TUs (A-type $=$, B-type $=, \mathrm{C}$-type $=$ ) in the specified peak hour are less due to the lower speed of some TUs during operation. Losses of kilometers due to losses in cycle time were calculated from the operational data. By inserting values of standard kilometers and actual kilometers for the individual type of rolling stock, we get the performance of the RS:

Performance $=\left[\left(0.62 \cdot \frac{1419}{1442.19}\right)+\left(0.195 \cdot \frac{417}{438.47}\right)+\left(0.185 \cdot \frac{408}{409.26}\right)\right] \cdot 100=97.99 \%$

As mentioned earlier, the total number of cars put in service during the one selected peak hour is 470 . Some of the cars suffer complete breakdowns during operation. Consequently, these TUs were withdrawn from or not admitted to service. According to the definition the quality yield becomes

Quality yield $=\sum_{n=1}^{470}\left(\frac{n w}{n}\right) \cdot 100=\left[\sum_{n a=1}^{296}\left(\frac{n w a}{n a}\right)+\sum_{n b=1}^{90}\left(\frac{n w b}{n b}\right)+\sum_{n c=1}^{84}\left(\frac{n w c}{n c}\right)\right] \cdot 100$

Where, $n=$ the total number of cars put into service and $n w=$ the number of cars actually operated during operation time. There are three compositions; therefore, $n=n a+n b+n c$ and $n w=n w a+n w b+n w c$. After inserting the values, we get

Quality yield $=\left(\left[0.63 \cdot \frac{295}{296}\right]+\left[0.1914 \cdot \frac{89}{90}\right]+\left[0.1787 \cdot \frac{84}{84}\right]\right) \cdot 100=99.57 \%$ 
An Approach to Calculate Overall Efficiency of Rolling Stock for an Urban Rail Transit System

Finally, the OEE of the RS as a product of availability, performance and quality yield is

$$
O E E=(0.8958 \cdot 0.9799 \cdot 0.9957 \cdot 100)=87.40 \%
$$

\section{Discussion and Future Work}

The OEE measure can be used to calculate the overall efficiency of the RS and gives useful information. It measures the efficiency of the equipment during its planned operation time, while the planned downtime does not affect the OEE, no matter how long it is. Different inputs/outputs of the parameters have lead to different methods for their calculation; the flexibility of the OEE measure allows this. After the OEE measure has been obtained, any type of RS with low scores in parameters can be carefully studied to develop plans of action aiming to improve the OEE. In the case study above, availability of the RS is the main factor responsible for the lower OEE.

It is difficult to defend this percentage of OEE. There can be a number of values of the three OEE parameters that can all lead to the final result of 87.40 percent. The studies carried out worldwide by researchers indicate that the average OEE rate for any production plant is 60 percent, and an OEE percentage that is equal to or greater than 85 is considered a World Class OEE (Vorne 2002).

Nevertheless, these results are not applicable for the OEE of the RS. It is the task of URTS management to decide upon the OEE level of the rolling stock. The worth of gain in a single percentage of availability, performance, and quality yield should be linked with their objectives and requirements. For example, one agency gives priority to availability at the cost of an acceptable reduction in performance, whereas a second agency does not compromise on performance. The success and failure criteria for the OEE and its parameters for a particular rolling stock of a URTS vary from system to system and represent the priorities and limits of that system.

The OEE cannot be used to rank two RS that differ in availability, performance, and quality yield but have the same OEE percentages. In this case, it would be highly subjective to differentiate the overall efficiency of two rolling stocks with different characteristics. One way to deal with this problem is to define standards for the OEE and its parameters for different URTS and their subunits. These standards will work as a reference for the comparison of different URTS with similar characteristics.

There may be discussion about the percentage point improvement in availability, performance, or quality yield and whether it is worth improving the OEE. What 
is the acceptable percentage of availability, performance, and quality yield for a specific URTS? Similarly, what percentage of OEE is better for which type of rolling stock, and what type of rolling stock is technically suitable for future service? Such questions are part of modeling decision problems regarding the OEE and its parameters for a particular URTS and could be part of future work. The OEE measure is a simple and effective formula that can be used to easily handle the decision problems described. The flexibility of the OEE approach makes it applicable to other forms of public transport, provided that the inputs and outputs of the parameters of OEE exist. It is important that inputs and outputs of the three parameters of the OEE are defined clearly. If the definitions of the parameters are interrelated, then they could be measuring the same things more than once, which could lead to incorrect conclusions.

\section{Conclusions}

The overall efficiency of the rolling stock (RS) of urban rail transit system (URTS) was defined. Various types of inputs and outputs of the RS were considered for three parameters of the overall efficiency, and a method for their calculation was presented and illustrated by providing a real-world application. The simplicity, flexibility, and efficiency of the measure of overall efficiency make it applicable and useful for any kind of RS or other subunits of URTS where decision problems need to be solved.

\section{Acknowledgments}

The authors would like to thank Prof. Jochen Trinckauf of TU Dresden for his input to the research described in the paper. Financial support of the Higher Education Commission (HEC) Pakistan and the public transport company of Munich (MVG) is acknowledged.

\section{References}

Barnum, Darold T., S. McNeil, and J. Hart. (2007). Comparing the efficiency of public transportation. Journal of Public Transportation 10(2).

Borris, S. (2006). Total productive maintenance. In S. Borris, TP: Proven Strategies and Techniques to Keep Equipment Running at Peak Efficiency. New York: McGraw-Hill. 
An Approach to Calculate Overall Efficiency of Rolling Stock for an Urban Rail Transit System

Cooper, W. W., L. M. Seiford, and J. Zhu. (2004). Handbook on Data Envelopment. Boston: MA: Kluwer Academic Publishers.

Dhillon, B. (2002). Engineering Maintenance: A Modern Approach. Boca Raton, Florida: CRC Press.

Edwards, J. (1992). Transportation Planning Handbook. NJ: Prentice-Hall.

Levitt, J. (1997). Maintenance Management. New York: Industrial Press.

McCarthy, P. W. (2001). TPM. In TPM: A Route To World Class Performance. Oxford: Butterworth Heinemann.

Omdahl, T. (1988). In Reliability, Availability, and Maintainability (RAM) Dictionary. Milwaukee, Wisconsin: ASQC Quality Press.

Sulek, J. M., and M. R. Lind. (2000). A systems model for evaluating transit performance. In A Guide Book for Developing a Transit Performance-Measurement System, Transportation Research Board, retrieved April 15, 2011, from TCRP Report 88: http://onlinepubs.trb.org/onlinepubs/tcrp/tcrp_report_88/Guidebook.pdf

Vorne, I. (2002). World Class OEE. Retrieved April 10, 2011, from Fast Track OEE: http://www.oee.com/world_class_oee.html

VTPI. (2011). Online TDM Encyclopedia. Retrieved May 13, 2011, from Victoria Transport Policy Institute: http://www.vtpi.org/tdm/index.php

Vuchic, V. (2005). Urban transit. In V. R. Vuchic, Urban Transit: Operations, Planning, and Economics. Hoboken, New Jersey: John Wiley \& Sons.

\section{About the Authors}

M.Sc. QAMAR MAнвоOB (qamarmahboob.pk@gmail.com) earned a B.Sc. in Mechanical Engineering from UET Lahore, and M.Sc. in Total Quality Management from PU Lahore, and an M.Sc. in Transportation Systems from Technische Universität München (TUM). He has been with Pakistan Railways as a Maintenance and Planning Engineer since 2000. Presently, he is on study leave to work towards his Ph.D. in safety, risk and reliability in railway systems from the Technische Universität Dresden, Germany.

Dipl.-Geogr. Thом AS STOIBER (thomas.stoiber@gmx.net) studied geography at the University of Augsburg and worked as a researcher at Technische Universität 
München in the Department of Urban Structures and Transport Planning between 2007 and 2010. His primary activities were in the fields of accessibility planning and integrated spatial and transport development in public transport corridors. Since September 2010, he has worked for Ernst Basler + Partner AG, a planning consultancy in Zurich, Switzerland.

Dipl.-Ing. Stephanie GotTstein (gottstein.stephanie@swm.de) studied electrical engineering at the Technische Universität München (TUM). She has been KVPManager (continuous advancement process manager) since 2006 at Stadtwerke München $\mathrm{GmbH}$ (SWM), Department of Transport Services. She is responsible for rolling stock advancement and project management for rebuilding and deals with the problem of obsolescence in electronical spareparts.

Dr.-Ing. Antonios TsakARestos (tsakarestos@vt.bv.tum.de) studied civil engineering at the Technische Universität München (TUM). Since 2002, he has been a scientific assistant for the chair of Traffic Engineering and Control at TUM, where he is in charge of the research group for public transport systems. In 2010, he completed his Ph.D. on the methodology of public transport planning for rural areas. 